\title{
Mira Variables explained by a planetary companion interaction: A means to drop the pulsation paradigm?
}

\author{
P. Berlioz-Arthaud ${ }^{\star}$ \\ CRAL-Observatoire de Lyon, 9 Av. Charles André, 69561 Saint Genis Laval Cedex, France
}

Received 29 July 2002 / Accepted 30 September 2002

\begin{abstract}
We emphasize the limits of the classical paradigm explaining the periodic appearance of emission lines by shock waves induced by radial periodic pulsations. In particular, we argue that, if pulsation occurs, it would not be purely radial and the light curve resulting from the contribution of zones at different phases would have a shape different from what we actually observe. The discovery of low mass companions to numerous G stars, some of them very close to the star, led us to look at the fate and the influence of this companion when the star becomes a giant. After Rudnitskij (2000), we suggest that the behavior of the Mira variables is mainly due to the presence of a companion in the tenuous atmosphere of the giant instead of the effect of a pulsating atmosphere explaining the presence of emission lines and the periodicity of these variables. We argue that the presence of a companion may account for the shape of the light curve and the observed phase lag between the maximum visual and the maximum maser light.
\end{abstract}

Key words. stars: late-type - stars: oscillations - stars: variables: general

\section{Introduction}

Following the main sequence evolution, a low-mass star (3-5 solar mass), experiences a somewhat complicated sequence (Iben \& Renzini 1983) of adjustments owing to the passage from hydrogen- to helium-burning. After a preliminary phase where the star is burning helium in a shell around its core (early asymptotic giant branch, E-AGB) energy is mainly produced by hydrogen burning in a shell. As the luminosity increases, the star inflates and its surface temperature lowers: high luminosity and low temperature locate these stars in the top left of the HR-diagram, along the so-called asymptotic giant branch (AGB). At the end of their evolution these stars expel their external envelope leading to planetary nebulae with hot white dwarfs in their center.

The General Catalog of Variable Stars (GCVS, Kholopov et al. 1988) records with certainty 5277 Miras, 877 type A (SRa) and 1175 type B (SRb) semiregular variables. These two last types are only distinguished from Miras by their smaller amplitude (less than $2.5 \mathrm{mag}$ against 2.5 to 11 for Miras) or period or their less regular light curve. Moreover 1725 type B irregular variables $(\mathrm{Lb})$ are listed. All these objects fall into the "pulsating variables" type.

Many attempts have been made at deducing the main characteristics of these stars (mass, luminosity, radius) from the characteristics of their light curves (period, amplitude, color).

^ e-mail: pberlioz@obs.univ-lyon1.fr
To account for the variations of light, the appearance of emission lines near the maximum, and the variable velocities of the absorption and emission lines, a scenario progressively came out: the star experiences radial periodic pulsations, the emission lines appearing when an expanding shock, induced by the pulsation, emerges from the photosphere. According to this view, the periodic behavior of the star reveals the periodic pulsation.

Due to the difficulties, work in this field has been carried out in different ways; we quote some of them:

- analysis of many spectra of Miras variables (Joy 1926; Merrill 1946, 1947, 1953; Deutsch \& Merrill 1959; Maehara 1968; Tsuji 1971);

- development of pulsation theory (Eddington 1941, 1942; Baker \& Kippenhahn 1962; Zhevakin 1963; Fox \& Wood 1982);

- dynamics of atmosphere (Willson 1976; Willson \& Hill 1979; Bowen 1988; Feuchtinger et al. 1993);

- shock wave models (Fox \& Wood 1985; Gillet 1988).

In spite of many attempts, we are far from having a satisfactory understanding of these objects. In particular, we are not able to link, in a consistent way, models of pulsation with models of atmospheres, or models of atmospheres with shock waves models. Thus, so far, it has not been possible to give a qualitative interpretation of the outstanding features of these spectra.

Joy (1926) looked at the binarity to explain the velocity curve of the absorption lines of Mira: he derived an elliptic 
orbit with a semi-axis projected on the sky of $16200000 \mathrm{~km}$ that he found to be far inside the known size of the star, and this idea has not been followed up.

Rejecting the pulsation hypothesis, we found that the presence of a companion in the atmosphere would be able to explain the observed properties of Miras. A year later, this paper being almost fully written, we knew of a poster (Rudnitskij 2000) proposing, two years before us, the same explanation. We chose to maintain the whole argumentation we independently conceived in order to show similarities and eventually differences of the two approaches. We only modified this introduction, the title, the order of sections, emphasizing the origin of our work in the limits and failures of the pulsation theory rather than in the fate of planets in the atmosphere of AGB stars.

In his poster, Rudnitskij (2000) discusses the effect of a low-mass companion revolving around a red giant inside its circumstellar envelope. He quotes Soker (1999) examining the effect of a planet inside planetary nebulae which may lead to the formation of a tail strongly emitting in $\mathrm{H} \alpha$. Rudnitskij considers that Jupiter-like planets can survive a significant time during the AGB stage at a distance of 1 AU from the star and would produce a conical shock. The shock may add light variations and account for the observed emission lines. Regarding semiregular variables, Rudnitskij interprets these stars as having less massive planets than Miras or being seen almost perpendiculary to their orbital plane. He proposes the effect of a close companion as an alternative to the pulsation-driven shocks mainly because of the weakness and the too low velocities of the observed shocks with respect to what is deduced from pulsation theory. Finally, Rudnitskij concludes that his model may explain the asymmetry in brightness distribution, the light curve, and the velocity structure of emission lines (e.g. doubling).

In Sect. 2, we emphasize the objections to the pulsation theory, in Sect. 3 we examine the consequences of the axisymmetry, in Sect. 4 the fate of a companion in the atmosphere of a giant, in Sect. 5 the effect of a companion in the atmosphere of AGB stars, in Sect. 6 we search after observable properties able to discard one explanation, and in Sect. 7 we reconsider the interpretation of the features of masering source found around Miras and semiregular variables before concluding.

\section{Are Miras pulsating variables?}

The classical explanation of the variability of Miras is based upon the periodic development of shock waves appearing below the photosphere and expanding outward. These shocks may explain the emission lines. To explain the periodicity of the variability, the shock waves are supposed to be induced by a pulsation. This pulsation must be radial, i.e. it must affect the whole sphere of the star. This condition is necessary to explain the shape of the light curve with a well-marked main period. Non radial modes should produce a light curve with several out-of-phase variations different from that we actually observe.
Two main arguments that we develop hereafter, may discard a purely radial pulsation:

1. if pulsation occurs, it is unlikely that it should be radial;

2. purely radial pulsation and convection are not compatible.

\subsection{Non radial modes}

Mira variables are supposed to be radial pulsators, which means that the pulsation is synchronized on the whole sphere of the star. But, if pulsation occurs, we must expect that a nonradial mode would be with certainty excited because during a pulsation period a sound wave starting somewhere inside the star cannot reach simultaneously the whole surface of the star but only a limited part of the pulsating sphere, making a pure radial pulsation unlikely. Thus the growing of two perturbations sufficiently far from each other can lead to two neighboring zones having opposite phases.

The fact that the only possible driving mechanisms is located into the ionization zone of hydrogen or helium (see hereafter), i.e. at about $20 \%$ of the radius of the star below its surface (not at the center) strengthens this argument.

\subsection{Coupling between pulsation and convection}

A further difficulty is linked to the highly convective envelope of late-type giant stars. Schwarzschild (1975) showed that a maximum of 200 and maybe as few as a half-dozen convective cells occupied the visible half of a red giant. He estimated an average convective velocity of the order of the sound velocity $\left(5 \mathrm{~km} \mathrm{~s}^{-1}\right)$ and a time scale for convection of $\sim 200$ days. Even if these estimates are not accurate, the motion of convective cells will again disturb the propagation of the pulsation leading to a phase lag between neighboring area and hence non radial pulsation.

Zhevakin (1963) claims that "a zone with convective energy transfer cannot maintain stellar auto-oscillations" and thus explains why dwarfs and sub-dwarfs cannot pulsate. As a consequence, he asserts that "in spite of the convective instability, $[. .$.$] radiative energy transfer predominates over convective$ transfer". This last argument is difficult to accept: model atmospheres in hydrostatic equilibrium show that $90 \%$ of the flux is still transported by convection at $95-97 \%$ of the radius (e.g. Plez 1992).

Moreover, energy transfer along a cycle of pulsation is another doubtful process: Mira variables have at least 0.7 mag of bolometric variations (Habing 1996), that is to say at least a luminosity ratio of 2 between maximum and minimum light. Assuming a period of 400 days and a luminosity of $7000 L_{\odot}$, the energy to be stored for half a cycle is $\sim 2 \times 10^{44} \mathrm{erg}$. If a $(\kappa)$-mechanism is invoked to explain pulsation, this energy must be stored as ionization energy. The mass to be ionized is $\sim 0.01 M_{\odot}$. The ionization zone lies between 5 and $20 \%$ of the stellar radius below the surface: the conditions of heat transfer have to change in such a large region for a time of 200 days: it is unlikely that the efficiency of convection will drastically change in a so short time. Around the time of minimum, with increasing ionization, we expect that convection would be enhanced. Is there a mechanism able to inhibit convection? 


\subsection{Pulsation modes and driving mechanism}

Other difficulties about the pulsation of Miras are well known:

- the pulsation mode (fundamental or overtone) is debated;

- the driving mechanism of the pulsation is, at this time, unclear.

Pulsation studies have been performed in order to derive the period-mass-radius relation: this relation is obviously different according to the mode of pulsation (fundamental or first overtone). This question was not answered conclusively in the course of IAU Symp. 191 (1999): there was evidence for the first overtone for Miras and semiregular variables (Feast 1999), at the same time observations were found to be compatible with the fundamental mode (Tuchman 1999), and a survey of the Large Magellanic Cloud led to the identification of Miras as radial fundamental mode pulsators and semiregular variables as fundamental, first, second or third-overtone pulsators (Wood et al. 1999).

Regarding the driving mechanism, Gautschy \& Saio (1996) reviewing the stellar pulsations ascribe it to the combined action of partial $\mathrm{H}$ and $\mathrm{He}$ I ionization but they stress that "energy transport by convection dominates", and that "the timescale of convective overturn is of the same order as the pulsation cycle. Hence, any statements on the pulsational driving and on the extension of the instability region depend crucially on our understanding of the coupling of pulsation and convection [...]. Both aspects are not well comprehended at present".

\section{Spherical symmetry versus axisymmetry}

Barring clear evidence to the contrary, astrophysical objects are normally assumed to be spherical. Interferometric observations make it possible to obtain a 2D-power spectrum as far as phase information is available which needs one sufficiently large telescope or three telescopes and good signal-to-noise. In most cases, only diameters are available from interferometric measurements. According to this view, any variation of the object is thought of as affecting its whole surface simultaneously with central symmetry: physical conditions are the same on a sphere of given radius and all displacements are radial.

By contrast, many planetary nebulae appear to be axisymmetric spherically symmetric, rather than. Many studies (Morris 1981; Eggleton 1990; Soker 1997 and references herein) explain these structures by the presence of companions (planets, brown dwarfs or stars). Furthermore, many recent observations show disk-like structure around red giants and Miralike AGB stars: a dust disk is observed (Molster et al. 2001) around the carbon star IRAS 09425-6040. A rotating disk is suspected around an AGB star (Bergman et al. 2000). The presence of a ring of material needs a progenitor: a planet or a group of planets are indispensable progenitors of the disks and rings observed.

Furthermore, companions have been invoked to explain the following:

- fast rotating horizontal branch stars (Soker \& Harpaz 2000);
- the presence of SiO masers (Struck-Marcell 1988);

- the oxygen-rich circumstellar envelopes of carbon stars (Barnbaum et al. 1991);

- the rotation of V Hya (Knapp et al. 1999).

\section{The fate of planets around red giants}

Since the first discovery (Mayor \& Queloz 1995) of an exoplanet around a star in 1995, already 87 stars (http://www.obspm.fr/planets/) have been shown to possess one or several planets leading us to think that it is a common situation. During the AGB stage, the luminosity rises to about $10^{4} L_{\odot}$ and the radius of the star increases. Lunar occultation and interferometric methods make it possible to measure visibility functions of Miras and semiregular variables. To convert these visibility functions into linear radii, one needs to know distances and limb darkening which are not well known. The resulting linear radii are all the more uncertain as they may vary with phase and wavelength. Thus the results for 18 Mira variables (van Belle et al. 1996) range from 224 to $580 R_{\odot}$ and for 10 Mira variables other observers (Haniff et al. 1995) derived radii between 392 and $606 R_{\odot}$. With this range of radii, a planet around a star of 1-1.5 solar mass will orbit near the photosphere if its period is in the range 320-780 days. The actual range of periods of the sample is 284 to 430 days: considering the uncertainties, planet interaction is not excluded.

Wood et al. (1999), analyzing MACHO observations of LMC red giants, find that perhaps $\sim 25 \%$ of AGB stars are semidetached or contact binary systems: they identify contact binaries lying on the first giant branch (their sequence $\mathrm{E}$ ) with periods ranging from 30 to 250 days and stars with "long secondary periods (LSP)" (their sequence D) ranging from $\sim 150$ to 1200 days. For this last sequence, Wood et al. (1999) propose that "the LSPs are due to an eclipse of the AGB star by a cloud of dusty accreted matter surrounding an orbiting, invisible companion star". Wood et al. (1999) do not think these stars are pulsating variables, because they lie on a period-luminosity sequence with periods greater than that derived from fundamental pulsator models: only shorter periods may be explained by higher harmonic modes. The only way to mark out pulsating variables and binaries is from their periods. But we must suppose that many stars belonging to the Mira sequence also have near companions. In particular, what happens to sequence E stars when they reach the AGB with orbital periods in the range of that of Miras?

What one wants to know is if the planet will survive, close to the star, for a length of time of the order of the Mira phase $\left(\sim 10^{5}\right.$ years). One argument (Lin et al. 1996) to explain the survival of the companion of $51 \mathrm{Peg}$ at $0.05 \mathrm{AU}$ from the star is based upon the comparison of the escape velocity at the surface of the planet and the thermal velocity of hydrogen atoms: from the surface of a planet similar to Jupiter, the escape velocity is $\sim 62 \mathrm{~km} \mathrm{~s}^{-1}$ and the mean thermal velocity of hydrogen atoms at the effective temperature $(2200 \mathrm{~K})$ of the star is $\sim 6 \mathrm{~km} \mathrm{~s}^{-1}$ leading to a negligible mass loss during the AGB phase. Moreover, the point where the gravitational attraction from the planet and the star are equal is $\sim 0.03 \mathrm{AU}$ from the center of the planet ( $\sim 65$ planet radii) and the inner 
Lagrangian point is located at $\sim 0.07$ AU from the planet center $(\sim 150$ planet radii): the planet will not fill its Roche lobe and no matter will escape this way to the star.

One important element of the system evolution is the angular momentum conservation. The moment of inertia is estimated by Spruit (1998):

$I=k^{2} M R^{2}$

with $k^{2}$ being $\sim 0.2$ for an AGB envelope, $M$ and $R$ the mass and external radius of the envelope.

Thus, assuming that the variation of angular momentum of one Jovian mass shifted from an orbit of 5.2 to $1 \mathrm{AU}$ is deposited in an envelope of $0.1 M_{\odot}$ in rigid rotation and with an external radius of $1 \mathrm{AU}$, the envelope initially at rest would have a period of rotation as a solid body of 16 years. In the same way, the angular momentum of a planet of $\sim 16$ Jovian mass shifted from the orbit of Jupiter to the external radius of the AGB star, would be sufficient to enhance the rotation rate of the envelope to such a value that the external equatorial surface would be Keplerian. In conclusion, the orbital momentum of a planet with a mass equal or greater than a Jovian mass, deposited into the envelope of an AGB star produces a rotation of a part of the envelope eventually close to a Keplerian rotation.

Various physical mechanisms have been examined to explain the transfer of angular momentum inside a binary system (Zahn 1977) from the rotation of the more massive component to the orbital motion of the other one: tidal forces are invoked which create a torque between the primary rotating star and the secondary orbital motion - until synchronization occurs, i.e. when the period of rotation of the primary is the same as the orbital period. The rate of angular momentum transfer (and thus the characteristic time of this exchange), will depend upon the dissipation rate of the tidal perturbation in the envelope of the primary, mainly related to the viscosity of the envelope. The efficiency of such mechanisms is poorly known, evaluations (Zahn 1977) give a characteristic time much greater than the duration of the AGB phase. Anyhow, if this time is so long that the planet would not move for the duration of the AGB phase, the recent discoveries show many planets at least as massive as Jupiter the orbits of which are inside the radius of an AGB star. If this time is shorter than the AGB life time, there would be many planets with sufficient mass and distance to deposit angular momentum in the envelope such as to obtain synchronization, thus stopping the exchange of angular momentum.

The main mechanism of angular momentum exchange is probably due to the drag force on the planet in the atmosphere. This may be evaluated through the momentum exchange rate due to accretion onto the planet. The radius $R_{\text {acc }}$ where the momentum exchange due to gravitational force exerted on the gas is equal to the momentum of the gas is:

$R_{\mathrm{acc}}=\frac{G M_{\mathrm{P}}}{v^{2}}$

with $G$ the gravitational constant, $M_{\mathrm{P}}$ the mass of the planet, and $v$ the velocity of the planet. Following our example of a Jovian planet at $1 \mathrm{AU}$ from its star, we find $R_{\text {acc }} \sim 2$ Jovian radii.
Assuming that all the gas swept up in this radius is accreted on the planet leads to an accretion rate $M_{\text {acc }}$ :

$M_{\mathrm{acc}}=\frac{\pi\left(G M_{\mathrm{P}}\right)^{2}}{v^{3}} \rho$

with $\rho$ the medium density.

We estimate the characteristic accretion time as:

$t_{\mathrm{acc}}=\frac{M_{\mathrm{P}}}{M_{\mathrm{acc}}}$.

With a planet of one Jovian mass orbiting at $1 \mathrm{AU}$ around an AGB star of $1 M_{\odot}$, a characteristic time of $10^{5}$ years corresponds to a density of $\sim 10^{-10} \mathrm{~g} \mathrm{~cm}^{-3}$, close to the density of a red giant near the photosphere.

Some Miras show clear period decreases: Whitelock (1999) quotes R Aql (1.0 day per year) and R Hya (1.3 day per year) which gives for both cases a density of $\sim 10^{-8} \mathrm{~g} \mathrm{~cm}^{-3}$. This would locate the planet inside the photosphere for these extreme examples.

\section{Effect of a planet inside a red giant star atmosphere}

The passage of a companion in the tenuous atmosphere of the AGB star will induce tidal forces and movements leading to wave shocks owing to the relative slowness of the sound speed in such an atmosphere $\left(\sim 5 \mathrm{~km} \mathrm{~s}^{-1}\right)$ with regard to the velocity of the companion and its associated perturbation $\left(\sim 30 \mathrm{~km} \mathrm{~s}^{-1}\right)$.

This interaction with the stellar atmosphere will probably enhance the local temperature leading to a reduction of the opacity: thus the light curve of the star may be explained by a zone of reduced opacity passing through the stellar disk. We tried to reproduce the light curve: Fig. 1 shows the observed visual light curve of Mira (o Ceti) for two successive cycles drawn together with a synthetic light curve adjusted according to:

$F_{\phi}=\int_{\phi-\frac{\pi}{2}}^{\phi+\frac{\pi}{2}} I_{\theta} \cos (\theta+\phi) \sin i \exp \left(\frac{-\tau_{\theta}}{\cos (\theta+\phi) \sin i}\right) \mathrm{d} \theta$

with:

$F_{\phi}$ flux received from star at phase $\phi$;

$\phi$ phase of the light curve in radian;

$\theta$ longitude on the star;

$I_{\theta}$ intensity at longitude $\theta$;

$\tau_{\theta}$ optical depth at longitude $\theta$;

$i$ inclination of the orbit plane on the sky.

A finer tuning of parameters is limited by the scatter of the data inside a cycle and the cycle to cycle variations of the actual light curve of Mira (evident from the differences between successive maxima). We don't claim this simple model accounts for the complex interaction between the atmosphere and the companion but we only want to show there is some way to explain the light curve shape.

Another consequence is that the inclination of the orbit on the sky being randomly distributed, the amplitude would be reduced with increasing inclination giving an explanation of the 


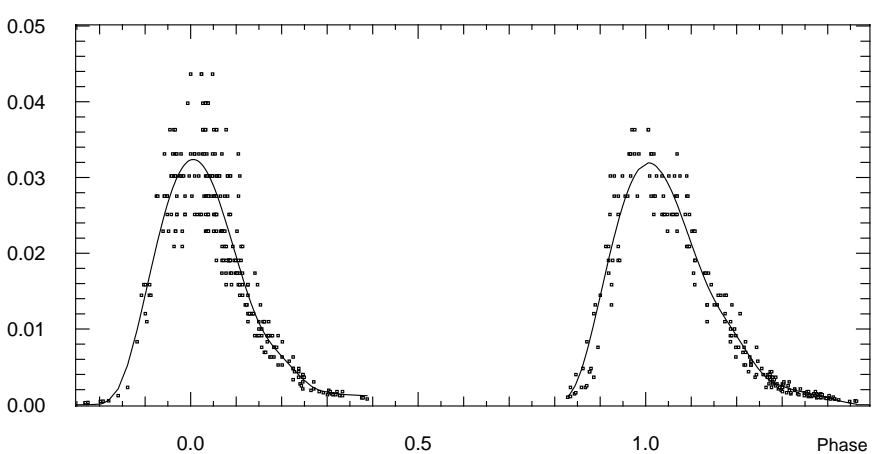

Fig. 1. Light curve of o Ceti (dot) as a function of phase for two successive cycles and synthetic light curve (line). Ordinates are fluxes (not magnitudes) on an arbitrary scale. (Data from Association Francaise des Observateurs d' Etoiles Variables AFOEV).

bad correlation between amplitude variation and mass-loss and a possible link between Miras, semiregular (of same period) and irregular variables; possibly these last stars are viewed pole-on.

Figure 2 shows detailed profiles of Balmer emission lines of $\chi$ Cygni at three different phases obtained at the William Herschel Telescope in March, June and July 2000. These lines reveal at least three components of different velocities: the difference between extreme velocities of $\sim 52 \mathrm{~km} \mathrm{~s}^{-1}$ may be explained by the rotation of matter. A general trend is the displacement of the blue component with advancing phase. This effect may be similar to what is observed around some cataclysmic variables during their quiescent phases. These systems show hydrogen emission lines whose features are supposed to originate in a rotating disk of matter: $\mathrm{H} \alpha$ and $\mathrm{H} \beta$ emission lines profiles exhibit two extreme peak whose positions are constant throughout an orbit and a central peak with a sinusoidal evolution tracing a hot spot corotating with the companion (Mennickent et al. 2001; Skidmore et al. 2000; Mason et al. 2000). Due to the relative slowness of matter around a Mira $\left( \pm 20\right.$ to $50 \mathrm{~km} \mathrm{~s}^{-1}$ ), the effects on the emission lines are much less noticeable than for cataclysmic variables $( \pm 200$ to $800 \mathrm{~km} \mathrm{~s}^{-1}$ ) and are perhaps complicated by overlying absorption and turbulent motions. Nevertheless many aspects would be comparable. The more so as these cataclysmic variables have probably experienced a Mira phase in the past.

The same kind of explanation may account for the doubling of absorption lines around maximum constated by Hinkle (1978) or Alvarez et al. (2001).

Recently Struck et al. (2002 presented numerical hydrodynamical models of planets in Mira winds. They examined the effect of a planet in the wind of a pulsating Mira. They estimate the luminosity of the accretion burst to explain the shorttimescale optical flashes from Miras. They stress the complexity of the accretion process through, for example, the possible coupling of the partially ionized gas and the magnetic field.

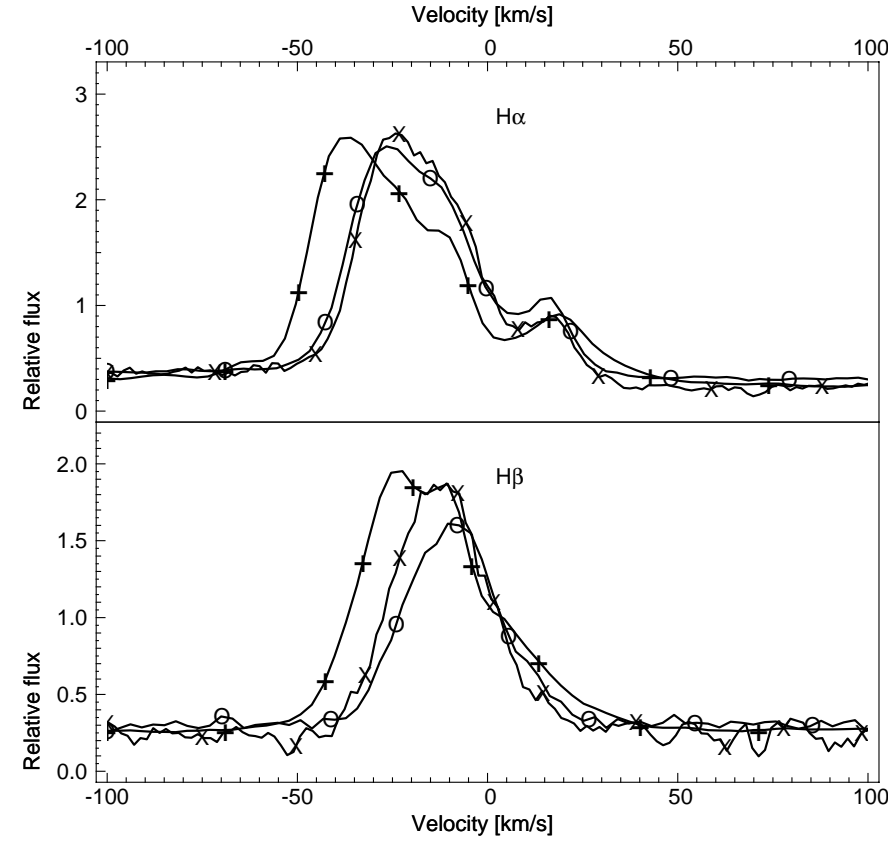

Fig. 2. $\mathrm{H} \alpha$ and $\mathrm{H} \beta$ lines of $\chi$ Cygni at three different phases: $0.22(+)$, $0.54(\times)$ and $0.58(\mathrm{o})$ assuming an heliocentric velocity of $7.5 \mathrm{~km} \mathrm{~s}^{-1}$. Fluxes are scaled to be comparable.

\section{Comparison with the observable properties deduced from the pulsation theory}

What observations allow us to decide between the two explanations? There are actually no observations that show the pulsation of Miras unequivocally.

In order to see whether some arguments may discard the explanation by a companion interaction, we find only two which are directly related to a pulsation: the period - mean density relation and the behavior of masers.

\subsection{Period - mean density relation}

No observations are directly related to the pulsation (e.g. radius versus phase), and only global relations between period, mass, radius and luminosity are available.

If the companion is close to the photosphere of the star, there is a relation between the mass $M$, the radius $R$ of the star, and the orbital period $P$ :

$P=\frac{2 \pi}{\sqrt{G}} \sqrt{\frac{R^{3}}{M}}$

with $G$ the gravitational constant.

This relation is similar to the period - mean density relation yielded by the pulsation theory with a pulsation constant $Q$ :

$$
P=Q \sqrt{\frac{\rho_{\odot}}{\rho}}
$$

with $\rho_{\odot}$ and $\rho$ the mean densities of the sun and the star respectively.

In the case of a companion interaction, $Q$ has a constant minimal value of 0.116 day for a companion orbiting at the radius of the star or beyond. By contrast, in the case of a 
pulsation, the value of $Q$ varies with the mode of pulsation and the structure of the star. From theoretical models, Fox $\&$ Wood (1982) derived pulsation constants for $1 M_{\odot}$ models from 0.077 to 0.114 day with periods and luminosities ranging from 175 to 666 days and from 2510 to $6310 L_{\odot}$ respectively. For the first overtone, they found pulsation constants ranging from 0.039 to 0.046 day and periods from 90 to 260 days. Non adiabatic pulsation calculations led Gautschy (1999) to favour the fundamental mode for Miras with a value of $Q$ from 0.085 to 0.095 for $1 M_{\odot}$ models with periods of 260 and 380 days respectively - whereas for the first overtone, $Q$ is found to be about 0.04 day.

Thus we must expect that the qualitative relations established in the frame of the pulsation theory are valid in the case of a companion interaction - and the numerical values are expected to be close to those obtained for the fundamental mode.

\subsection{Maser}

Many semiregular and Mira variables are associated with maser emission from hydroxyl, water and silicon oxide molecules. $\mathrm{OH}$ masers are observed at three wavelengths: the $1612 \mathrm{MHz}$ satellite line, and the 1665 and $1667 \mathrm{MHz}$ main lines. Some of the maser source variations are related to the variations of the visual light curve. For the $1612 \mathrm{MHz}$ line two peaks in the spectrum are frequently observed and interpreted as a blue, approaching maser and a red, receding maser. Also a delay has been observed between the maximum of the blue and the red features (Reid \& Muhleman 1977; Herman \& Habing 1985). The general trend is that the blue peak maximum occurs before the red one.

The "thin-shell" standard model (Reid \& Muhleman 1977) for the $\mathrm{OH} 1612 \mathrm{MHz}$ maser comes into conflict with the explanation invoking a companion because it supposes that light variations are simultaneous on the whole surface of the star. Thus, in the following section, we will propose another explanation.

\section{Interpretation of maser phase lag}

In the classical interpretation (Reid \& Muhleman 1977), the blue peak comes from the approaching side of a thin expanding shell while the red peak comes from its receding side. The observed phase lag is explained by the light travel time to reach the opposite side of the shell and come back to the observer (Fig. 3). The diameter of the shell is found to be $\sim 8 \times 10^{15} \mathrm{~cm}$ ( $\sim 500$ AU) for Mira variables (Herman \& Habing 1985). This interpretation is questionable:

- 1665-1667 MHz OH maser main lines appear to be formed in a shell near the $\mathrm{H}_{2} \mathrm{O}$ maser (Szymczak et al. 1999; Masheder et al. 1999) at a radius of $\sim 50 \mathrm{AU}\left(8 \times 10^{14} \mathrm{~cm}\right)$, far inside the radius of the shell deduced from phase lag;

- 1665-1667 MHz OH or $\mathrm{H}_{2} \mathrm{O}$ maser components bluer than the $1612 \mathrm{MHz} \mathrm{OH}$ blue peak (supposedly originating from the outer accelerated shell) have been observed (Szymczak et al. 1999; Masheder et al. 1999; van Langevelde et al. 2000);

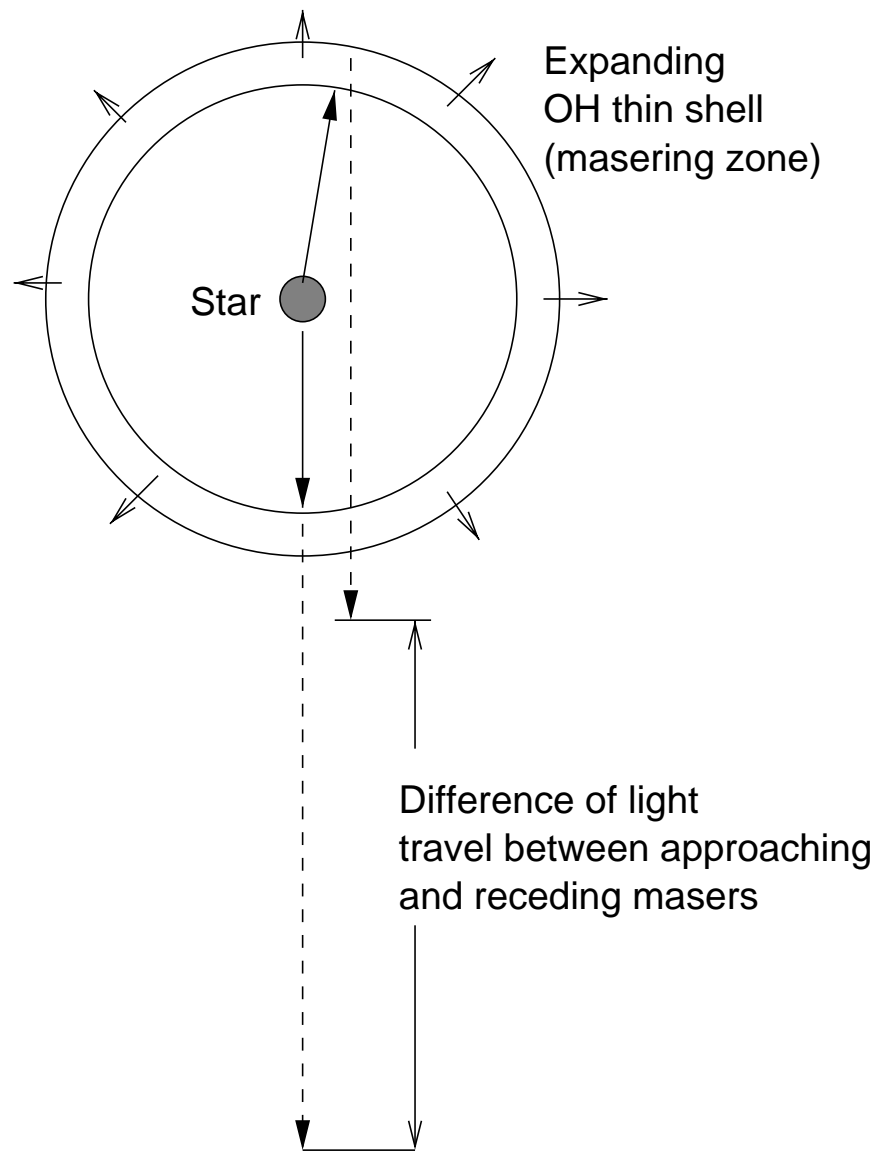

Fig. 3. Scheme of the standard expanding thin shell model. For an observer, the time of maximum of the receding (red-shifted) maser is delayed after that of the approaching (blue-shifted) maser. The diameter of the shell is deduced from the delay between the two maxima.

- the intensity of the most blue-shifted spot is not always the brightest (van Langevelde et al. 2000) whereas it should be amplified by the photosphere of the star;

- radial velocities deduced from visible absorption lines are red-shifted with respect to the velocity deduced from the $1612 \mathrm{MHz}$ OH maser mean velocity (Barbier et al. 1988).

Thus, if the expanding thin shell model is no longer valid, the phase lag between the blue and red $1612 \mathrm{MHz} O H$ maser peaks cannot be explained.

We account for these observations as follows: at maser maximum light the pencil of light from the star encounters the masering region near the ecliptic plane. The phase lag is deduced from the rotation of the bright zone of the star. Due to this view if the masering region is rotating around the star, the maximum of approaching (bluer) masers will be seen before the maximum of receding (redder) masers (Fig. 4). This conception is supported by high resolution maps showing a systematic displacement of the blue- and red-shifted emissions of the OH (Szymczak et al. 1999), and SiO (Hollis et al. 2000) masers. The rotation of a bright spot would produce a phase lag of the amplifying maser depending on the distribution of matter around the star.

It might be objected that the "thin-shell" model explains the observed well-marked two symmetrical peaks of the $1612 \mathrm{MHz}$ 


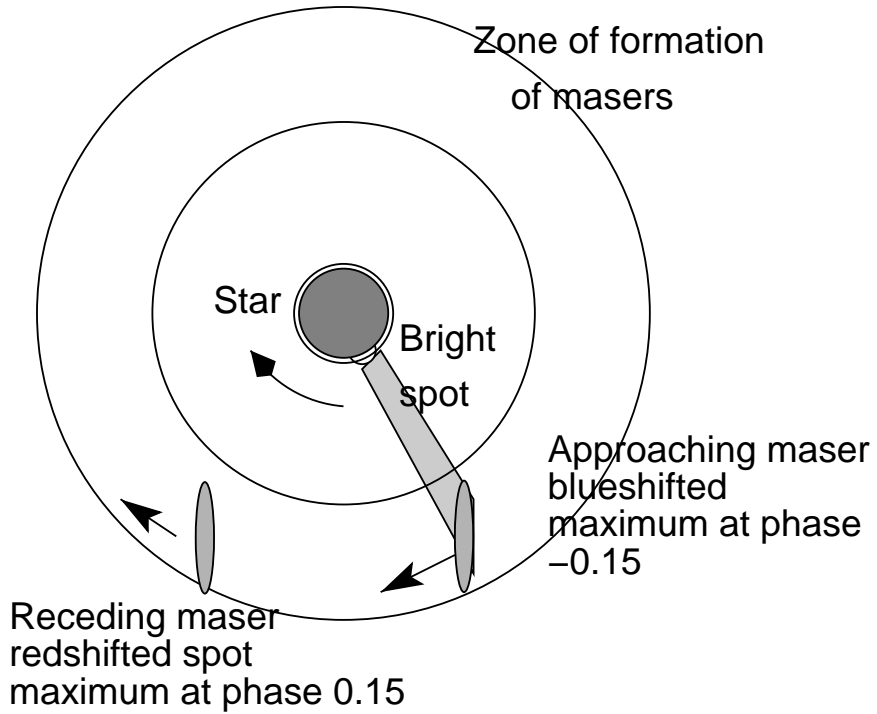

Fig. 4. Scheme of the rotating spot. The first illuminated maser is the approaching blue-shifted one. Thus the maximum of a blue-shifted maser spot appears before the maximum of a receding red-shifted maser spot.

emission. But we note that, while double peaks appear in $\mathrm{OH}-$ IR stars spectra, Miras and semiregular variables have irregular emission profiles with one or many peaks varying in velocity and intensity with time (see e.g. Etoka \& Le Squeren 2000).

\section{Discussion}

\subsection{Shock waves}

Willson \& Hill (1979) give examples of "considerable optical and infrared evidence supporting the existence of shock fronts in the stellar atmospheres":

1. emission lines and their behavior with phase (Willson 1976);

2. doubling and velocities of infrared lines (Hinkle 1978);

3. velocities of absorption lines (Merrill 1946; Tsuji 1971).

Merrill (1946), analysing spectra of R Leo, describes the evolution of emission and absorption lines around maximum, but attempts no explanation. Moreover, in another paper about the pre-minimum spectrum of R Leo, Merrill (1952) writes "thus the hypothesis of volume pulsation finds little support in the measured displacements of the dark lines. In view of the long period, a pulsation of moderate velocity amplitude is, of course, not precluded".

Willson (1976) identifies some fluorescent lines and their associated pumping transitions and deduces a discontinuity of velocity through the shock (from the wavelength difference between excited and exciting transitions) of between $40 \mathrm{~km} \mathrm{~s}^{-1}$ and $80 \mathrm{~km} \mathrm{~s}^{-1}$. The fluorescent lines are supposed to be formed in a tenuous atmosphere ahead of the shock and the exciters located near the shock, which seems to be confirmed by the sign of the velocity differences. Next, the most red-shifted part of the emission is supposed to be hidden by the star, explaining a shift of the central wavelength and making it possible to derive the shock velocity. Combining these elements, Willson (1976) concludes that the velocity of the shock decays as the inverse of the shock radius.

Hinkle (1978), observing $\mathrm{CO}$ and $\mathrm{OH}$ infrared absorption lines of R Leo, and their doubling around the maximum, claims that the red- and blue-shifted molecular absorption lines are formed above and below the shock respectively and that the line-forming region is on the order of $160 R_{\odot}$. Even with the large value of the radius of R Leo assumed by Hinkle (1978) $\left(\geq 700 R_{\odot}\right)$, it is $\sim 24 \%$ of the radius and Hinkle (1978) points out that effects of spherical geometry should be considered. However Hinkle (1978) notes the absence of P-Cygni profiles. Moreover $\mathrm{OH}$ and $\mathrm{CO}$ molecules would probably be dissociated through the shock and, in any case, should produce emission lines rather than absorption lines in the post-shock region.

Tsuji (1971) supports the existence of shock waves across the atmosphere. In order to explain the relative velocities and excitations of different group of lines, and their phase lags, Tsuji (1971) deduces a comprehensive structure of a Mira variable. From outside:

1. a circumstellar envelope where low excitation blue-shifted absorption lines are formed;

2. a "normal" atmosphere layer with "normal" red-shifted absorption lines;

3. a shock layer with emitting hydrogen lines;

4. a layer under the shock where high excitation molecular absorption lines are formed;

5. a layer responsible for the high-excitation blue-shifted absorption lines.

If this explanation makes it possible to interpret the radial velocities, it leads one to suppose that:

- the circumstellar envelope, the "normal" atmosphere layer, the shock and the molecular layer are all optically thin, as absorption lines are visible from the innermost layer;

- thus, for R And, assuming that line doubling lasts for at least $15 \%$ of a period, and that the shock velocity relative to the "normal" atmosphere is about $35 \mathrm{~km} \mathrm{~s}^{-1}$, the shock extends over $\sim 1.9 \times 10^{13} \mathrm{~cm}$ or $270 R_{\odot}$.

Assuming the value of the radius given by Tsuji (1971) (440 $R_{\odot}$ ), the "normal" atmosphere has to be optically thin over at least $\sim 0.6 R_{\star}$ below this external radius. Not only this is an improbable value, but also such a situation would produce characteristic line profiles (P-Cygni) which are not observed.

This last objection may be applied also to the three preceding papers because interpreting the emission lines velocities as radial shock velocities leads one to suppose that the shock extends over distances of the order of the radius of the star. Spherical geometry effects should then appear. Thus, we consider that there is no clear evidence supporting the existence of radial periodic shock waves in the atmospheres of Miras.

\subsection{Other pulsating variables}

It is noteworthy that new "non-radial pulsating variables" types have been progressively added to successive issues of the GCVS. 
Similar arguments to those developed here may lead one to reject radial pulsation for other pulsating variables such as Cepheids. We then have to find another mechanism to account for their variability. As explained, we believe the solution is to drop the spherical view and turn to a motion around an axis. A full discussion of this point is outside the scope of this paper. We just note that the value of the "pulsation constant" we obtained may apply to Cepheids too. This minimal value of 0.116 day is at least twice the value obtained by pulsation theory, therefore reducing the discrepancy between the mass obtained by evolution and the mass deduced from pulsation theory.

\section{Conclusions}

Habing (1996) gives some good reasons to study AGB stars: "the stars have sufficiently complex structure to make them challenging tests of our ingenuity" and because "they are good testing grounds for ideas and theories".

We have presented some strong arguments against the radial pulsation theory, and in support of an external influence; however, many problems remain unsolved. In particular, assuming companion interaction, the evolution of AGB stars will certainly depend upon the characteristics of the planetary system around it (masses, distances of the companions) and the interaction between the atmosphere and the companion - modeling such interaction is not easy.

The presence of companions around common stars drew the attention to the influence of such a presence on the evolution of the central stars: thus matter around a star plays a role as the young star emerges from the enshrouding dusty cocoon, and at the end of its life, influences its last transformation into a planetary nebula and a dying white dwarf - the companions keeping, in the meantime, the main part of the system angular momentum.

Acknowledgements. The author would like to thank P. J. V. Garcia for providing the spectra taken at the William Herschell Telescope and J. Katgert who kindly took time to correct my mistakes in the English language. This research has made use of the AFOEV data.

\section{References}

Alvarez, R., Jorissen, A., Plez, B., et al. 2001, A\&A, 379, 305

Baker, N., \& Kippenhahn, R. 1962, Z. Astrophys., 54, 114

Barbier, M., Petit, H., Mayor, M., \& Mennessier, M. O. 1988, A\&AS, 72,463

Barnbaum, C., Morris, M., Likkel, 1., \& Kastner, J. H. 1991, A\&A, 251,79

Bergman, P., Kerschbaum, F., \& Olofsson, H. 2000, A\&A, 353, 257

Bowen, G. H. 1988, ApJ, 329, 299

Deutsch, A. J., \& Merrill, P. W. 1959, ApJ, 130, 570

Eddington, A. S. 1941, MNRAS, 101, 182

Eddington, A. S. 1942, MNRAS, 102, 154

Etoka, S., \& Le Squeren, A. M. 2000, ApJS, 146, 179

Eggleton, P. P. 1990, in From Miras to Planetary Nebulae. Which path for stellar evolution?, ed. M. O. Mennessier, \& A. Omont (Éditions Frontières), 513

Feast, Y. 1999, Asymptotic Giant Branch Stars, ed. T. Le Bertre, A. Lèbre, \& C. Waelkens, IAU Symp., 191, 109
Feuchtinger, M. U., Dorfi, E. A., \& Höfner, S. 1993, A\&A, 273, 513

Fox, M. W., \& Wood, P. R. 1982, ApJ, 259, 198

Fox, M. W., \& Wood, P. R. 1985, ApJ, 297, 455

Gautschy, A., \& Saio, H. 1996, ARA\&A, 34, 551

Gautschy, A. 1999, A\&A, 349, 209

Gillet, D. 1988, A\&A, 192, 206

Habing, H. J. 1996, A\&ARv, 7, 97

Haniff, C. A., Scholz, M., \& Tuthill, P. G. 1995, MNRAS, 276, 640

Herman, J., \& Habing, H. J. 1985, A\&AS, 59, 523

Hinkle, K. H. 1978, ApJ, 220, 210-228

Hollis, J. M., Pedelty, J. A., Forster, et al. 2000, ApJ, 543, L81

Iben, I., \& Renzini, A. 1983, ARA\&A, 21, 271

Joy, A. H. 1926, ApJ, 63, 281

Kholopov, P. N., Samus, N. N., Frolov, M. S., et al., General Catalogue of Variable Stars, 4th Edition, vol. I-III, 1985-1988 (Moscow: Nauka Publishing House)

Knapp, G. R., Dobrovolsky, S. I., Ivezic, et al. 1999, A\&A, 351, 97-102

Lin, D. N. C., Bodenheimer, P., \& Richardson, D. C. 1996, Nature, 392, 57

Maehara, H. 1968, PASP, 20, 77

Masheder, M. R. W., van Langevelde, H. J., Richards, A. M. S., Greenhill, L., \& Gray, M. D. 1999, NewAR, 43, 563

Mason, E., Skidmore, W., Howell, et al. 2000, MNRAS, 318, 440

Mayor, F., \& Queloz, D. 1995, Nature, 378, 355

Mennickent, R. E., Diaz, M., Skidmore, W., \& Sterken, C. 2001, A\&A, 376, 448

Merrill, P. W. 1946, ApJ, 103, 275

Merrill, P. W. 1947, ApJ, 106, 274

Merrill, P. W. 1952, ApJ, 116, 337

Merrill, P. W. 1953, ApJ, 118, 453

Molster, F. J., Yamamura, I., Waters, L. B. F., Nyman, L. A., et al. 2001, A\&A, 366, 923

Morris, M. 1981, ApJ, 249, 572

Plez, B. 1992, A\&AS, 94, 527

Reid, M. J., \& Muhleman, D. O. 1977, AJ, 214, 60

Rudnitskij, G. M. 2000, The impact of large-scale surveys on pulsating star research, IAU Colloq. 176, ed. L. Szabados, \& D. W. Kurtz, ASP, 203, 384

Schwarzschild, M. 1975, ApJ, 195, 137

Skidmore, W., Mason, E., Howell, et al. 2000, MNRAS, 318, 429

Soker, N. 1997, ApJS, 112, 487

Soker, N. 1999, MNRAS, 306, 806

Soker, N., \& Harpaz, A. 2000, MNRAS, 317, 861

Spruit, H. C. 1998, A\&A, 333, 603

Struck-Marcell, C. 1988, ApJ, 330, 986

Struck, C., Cohanim, B. E., \& Willson, L. A. 2002, ApJ, 572, L83

Szymczak, M., Cohen, R. J., \& Richards, A. M. S. 1999, MNRAS, 304, 877

Tsuji, T. 1971, PASP, 23, 275

Tuchman 1999, Asymptotic Giant Branch Stars, ed. T. Le Bertre, A. Lèbre, \& C. Waelkens, IAU Symp., 191, 123

van Belle, G. T., Dyck, H. M., Benson, J. A., \& Lacasse, M. G. 1996, AJ, 112, 2147

van Langevelde, H. J., Vlemmings, W., Diamond, P. J., Baudry, A., \& Beasley, A. J. 2000, A\&A, 357, 945

Whitelock, P. A. 1999, NewAR, 43, 437

Willson, L. A. 1976, ApJ, 205, 172

Willson, L. A., \& Hill, S. J. 1979, ApJ, 228, 854

Wood, P. R., Alcok, C., Allsman, R. A., et al. 1999, Asymptotic Giant Branch Stars, ed. T. Le Bertre, A. Lèbre, \& C. Waelkens, IAU Symp., 191, 151

Zahn, J.-P. 1977, A\&A, 57, 383

Zhevakin, S. A. 1963, ARA\&A, 1, 367 